DRAFT VERSION JUNE 21, 2018

Preprint typeset using LTEX style emulateapj v. 5/25/10

\title{
A DETECTION OF WEAK LENSING MAGNIFICATION USING GALAXY SIZES AND MAGNITUDES
}

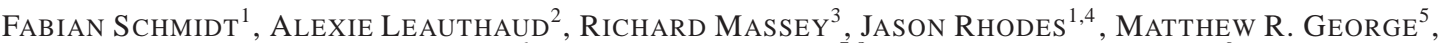 \\ Anton M. Koekemoer ${ }^{6}$, Alexis Finoguenov ${ }^{7,8}$, And Masayuki Tanaka ${ }^{9}$
}

Draft version June 21, 2018

\begin{abstract}
Weak lensing is commonly measured using shear through galaxy ellipticities, or using the effect of magnification bias on galaxy number densities. Here, we report on the first detection of weak lensing magnification with a new, independent technique using the distribution of galaxy sizes and magnitudes. These data come for free in galaxy surveys designed for measuring shear. We present the magnification estimator and apply it to an X-ray selected sample of galaxy groups in the COSMOS HST survey. The measurement of the projected surface density $\Sigma(r)$ is consistent with the shear measurements within the uncertainties, and has roughly $40 \%$ of the signal-to-noise of the latter. We discuss systematic issues and challenges to realizing the potential of this new probe of weak lensing.
\end{abstract}

Subject headings: gravitational lensing: weak — large-scale structure of universe — dark matter

\section{INTRODUCTION}

Weak lensing has emerged as a powerful tool in cosmology and astrophysics (e.g., Refregier (2003b); Munshi et al. (2008); Hoekstra \& Jain (2008); Massey et al. (2010); Schrabback et al. (2010)). It probes the underlying total matter distribution, and can be used to tie the observed distribution of galaxies to that of the dark matter, the dominant component of the cosmic matter budget. So far, weak lensing has primarily been measured using the shear, through the statistical correlation of galaxy ellipticities (see Bartelmann \& Schneider (2001) for a review). In addition, weak lensing magnification has been detected through its effect on the number density of a flux-limited sample (magnification bias; Scranton et al. (2005); Hildebrandt et al. (2009)). Shear $\gamma$ and convergence $\kappa$, which are related to the magnification $\mu$ through $\mu=\left[\left(1-\kappa^{2}\right)+|\gamma|^{2}\right]^{-1}$, so that $\mu=1+2 \kappa$ to linear order, measure different properties of the density field. For an azimuthally symmetric lens,

$$
\begin{aligned}
\gamma & =\frac{\Delta \Sigma}{\Sigma_{\mathrm{cr}}} ; \quad \kappa=\frac{\Sigma}{\Sigma_{\mathrm{cr}}} \\
\Sigma_{\mathrm{cr}} & =\frac{c^{2}}{4 \pi G} \frac{D_{s}}{D_{L} D_{L s}} \\
\Delta \Sigma & =\bar{\Sigma}(<r)-\Sigma(r),
\end{aligned}
$$

where $\Sigma(r)$ is the projected surface mass density of the lens, $r$ is the physical transverse distance on the lens plane, and $D_{s}, D_{L}, D_{L s}$ denote the angular diameter distance out to the

\footnotetext{
${ }^{1}$ California Institute of Technology, MC 350-17, 1200 East California Boulevard, Pasadena, CA 91125, USA

${ }_{2}$ Institute for the Physics and Mathematics of the Universe, University of Tokyo, Kashiwa 2778582, Japan

${ }^{3}$ Institute for Astronomy, Blackford Hill, Edinburgh EH9 3HJ UK

${ }^{4}$ Jet Propulsion Laboratory, California Institute of Technology, Pasadena, CA 91109, USA

${ }^{5}$ Department of Astronomy, University of California, Berkeley, CA 94720, USA

${ }^{6}$ Space Telescope Science Institute, 3700 San Martin Drive, Baltimore, MD 21218, USA

${ }^{7}$ Max-Planck-Institut für extraterrestrische Physik, Giessenbachstraße, 85748 Garching, Germany

${ }^{8}$ Center for Space Science Technology, University of Maryland Baltimore County, 1000 Hilltop Circle, Baltimore, MD 21250, USA
}

source, lens, and between lens and source, respectively. Note that the shear is proportional to the differential surface mass density $\Delta \Sigma$, while the convergence probes $\Sigma(r)$ itself. The different radial dependences of $\kappa$ and $\gamma$ can be used to break degeneracies between the lens mass and density profile shape (Rozo \& Schmidt 2010).

The convergence $\kappa$ has previously been measured using the observed number density of background sources. In a fluxlimited survey, the observed number density is given by

$$
n_{\mathrm{obs}}=\bar{n}\left[1+\delta_{g}+(5 s-2) \kappa\right],
$$

where $\bar{n}$ is the mean number density, $\delta_{g}$ denotes the intrinsic fluctuation in galaxy density, and $s=\partial \ln \bar{n} / \partial m_{\text {cut }}$ for a sharp magnitude cut $m_{\text {cut }}$. This can be generalized to surveys that are also limited by a size cut (Schmidt et al. 2009b). By cross-correlating foreground lenses with background sources widely separated in redshift, one can isolate this effect to measure $\kappa$. Note that the intrinsic fluctuations $\delta_{g}$ act as noise, and any residual physical correlation with the lenses (e.g., due to photo-z uncertainties) will contaminate the signal.

In this paper, we report on a detection of magnification around X-ray selected galaxy groups in COSMOS using galaxy sizes and fluxes. Related approaches have been proposed in Jain (2002); Bartelmann \& Narayan (1995); Bertin \& Lombardi (2006); see also Ménard et al. (2010) who used individual quasar magnitudes, and Huff \& Graves (2011). We rely on the observed properties of individual galaxies and do not use the number density of galaxies as done in magnification bias measurements. One key advantage of this technique is that the contamination due to residual physical correlation with the lenses is likely much smaller than in magnification bias measurements, due to the weaker correlation of galaxy sizes and luminosities with environment (Maltby et al. (2010); Nair et al. (2010); Croton et al. (2005)).

Throughout, we adopt a flat $\Lambda \mathrm{CDM}$ cosmology with $h=$ $0.72, \Omega_{m}=0.258$ to calculate distances. Halo masses are defined through a mean interior density of $200 \rho_{\text {crit }}(z=0.38)$, where $z=0.38$ is the mean redshift of the lens sample.

\section{MAGNIFICATION ESTIMATOR}

The data set we use consists of a set of values $\left\{d_{i}, m_{i}, z_{i}\right\}$ for each galaxy, denoting the size, magnitude, and photometric redshift. Let us consider how these observables change under 
lensing magnification. Throughout, we will work in the weak lensing regime. We can then write

$$
\begin{aligned}
d & =(1+\eta \kappa) d_{0} \\
m & =m_{0}+q \kappa \\
z & =z_{0} .
\end{aligned}
$$

Here, a subscript 0 denotes unlensed quantities, and in the last line we have assumed that photometric redshifts are not affected by lensing. In absence of noise and instrumental effects, the efficiency factors are $\eta=1$ and $q=-5 / \ln 10 \approx$ -2.17 , respectively (e.g., Schmidt et al. (2009b)). In practice, these values can be different due to the instrument PSF, and other effects and in general depend on the properties of a given galaxy.

In order to estimate $\kappa$ using Eqs. (5)-(6), we need to know the unlensed quantities $d_{0}, m_{0}$. These have some intrinsic distribution, and given the known distribution we can infer $\kappa$ statistically. Our starting point for the estimator is thus the joint distribution $p_{0}\left(d_{0}, m_{0} \mid z\right)$ of unlensed galaxy sizes and magnitudes at fixed redshift; the estimator is designed to not use the observed number density of galaxies. We approximate $p_{0}$ as Gaussian in $m$ and $x \equiv \ln d$. Furthermore, we approximate the error distributions in $m$ and $x$ as Gaussian. This greatly simplifies the evaluation of the estimator, at the price of making it sub-optimal. The log-likelihood is then given by

$$
\begin{aligned}
-2 \ln P(\Sigma) & =\sum_{i} \frac{1}{1-\rho^{2}\left(z_{i}\right)} \\
& \times\left\{\frac{1}{\sigma_{x, i}^{2}}\left(x_{i}-\bar{x}\left(z_{i}\right)-\tilde{\eta}_{i} F_{i} \Sigma\right)^{2}+\frac{1}{\sigma_{m, i}^{2}}\left(m_{i}-\bar{m}\left(z_{i}\right)-\tilde{q}_{i} F_{i} \Sigma\right)^{2}\right. \\
& \left.-2 \frac{\rho\left(z_{i}\right)}{\sigma_{x, i} \sigma_{m, i}}\left(x_{i}-\bar{x}\left(z_{i}\right)-\tilde{\eta}_{i} F_{i} \Sigma\right)\left(m_{i}-\bar{m}\left(z_{i}\right)-\tilde{q}_{i} F_{i} \Sigma\right)\right\} \\
\sigma_{x, i}^{2} & =\sigma_{x, \text { meas }, i}^{2}+\sigma_{x, \text { int }}^{2}\left(z_{i}\right)+\sigma_{x z, i}^{2} \\
\sigma_{m, i}^{2} & =\sigma_{m, \text { meas }, i}^{2}+\sigma_{m, \text { int }}^{2}\left(z_{i}\right)+\sigma_{m z, i}^{2} \\
\tilde{\eta}_{i} & =\eta\left(d_{i}\right)+\varepsilon_{x}\left(z_{i}\right) \\
\tilde{q}_{i} & =q\left(m_{i}\right)+\varepsilon_{m}\left(z_{i}\right) \\
F_{i} & =F\left(z_{i}\right)=\Sigma_{\mathrm{cr}}^{-1}\left(z_{i}\right) .
\end{aligned}
$$

The total variance in $x$ and $m$ is given by the sum in quadrature of the measurement uncertainty, intrinsic dispersion, and propagated photometric redshift uncertainty. $\sigma_{x, \text { meas }}, \sigma_{m \text {,meas }}$ are measured as a function of $d$ and $m$, respectively, through multiple images of the same galaxy from overlap regions of the HST ACS data (Leauthaud et al. 2007). The propagated redshift uncertainty is approximated as $(a=x, m)$

$$
\sigma_{a z, i}=\frac{1}{2}\left|\bar{a}\left(z_{+, i}\right)-\bar{a}\left(z_{-, i}\right)\right|,
$$

where $z_{ \pm, i}$ are the upper and lower $68 \%$ confidence level values for the redshift of galaxy $i$. The corrections $\varepsilon_{x}, \varepsilon_{m}$ to $\tilde{\eta}$ and $\tilde{q}$ take into account the fact that a positive magnification pushes faint, small galaxies over the flux and size thresholds (lensing bias). A similar effect exists for shear, but only at second order (Schmidt et al. 2009a), while the magnification estimator is affected at leading order. The correlation between intrinsic galaxy sizes and magnitudes is quantified by the correlation coefficient $\rho$.

The maximum-likelihood estimate $\hat{\Sigma}$ and its estimated vari- ance are then given by

$$
\hat{\Sigma}=\frac{\sum_{i} A_{i} F_{i}}{\sum_{i} B_{i} F_{i}^{2}} ; \quad \sigma^{2}(\hat{\Sigma})=\left(\sum_{i} B_{i} F_{i}^{2}\right)^{-1},
$$

where

$$
\begin{aligned}
& A_{i} \equiv \frac{1}{1-\rho^{2}\left(z_{i}\right)}\left\{\frac{\tilde{\eta}_{i}}{\sigma_{x, i}^{2}\left(x_{i}-\bar{x}\left(z_{i}\right)\right)+\frac{\tilde{q}_{i}}{\sigma_{m, i}^{2}}\left(m_{i}-\bar{m}\left(z_{i}\right)\right)}\right. \\
&\left.-\frac{\rho\left(z_{i}\right)}{\sigma_{x, i} \sigma_{m, i}}\left[\tilde{\eta}_{i}\left(m_{i}-\bar{m}\left(z_{i}\right)\right)+\tilde{q}_{i}\left(x_{i}-\bar{x}\left(z_{i}\right)\right)\right]\right\} \\
& B_{i} \equiv \frac{1}{1-\rho^{2}\left(z_{i}\right)}\left\{\frac{\tilde{\eta}_{i}^{2}}{\sigma_{x, i}^{2}}+\frac{\tilde{q}_{i}^{2}}{\sigma_{m, i}^{2}}-2 \frac{\rho\left(z_{i}\right)}{\sigma_{x, i} \sigma_{m, i}} \tilde{\eta}_{i} \tilde{q}_{i}\right\}
\end{aligned}
$$

The intrinsic dispersions $\sigma_{x, \text { int }}, \sigma_{m \text {,int }}$ as well as $\rho$ are determined by fitting a bivariate Gaussian distribution to all galaxies before cuts on $x, m$ in a redshift slice. We subtract the mean measurement error in $x, m$ in quadrature from the total measured dispersion to obtain $\sigma_{x, \text { int }}, \sigma_{m, \text { int }}$.

The quantities $\bar{x}, \bar{m}$ are determined by solving the equation $\hat{\Sigma}=0$ applied to all galaxies in a given redshift slice. Since $F \approx$ const within a narrow redshift slice, this yields

$$
\begin{aligned}
& \sum_{i} \frac{1}{1-\rho^{2}\left(z_{i}\right)}\left\{\frac{\tilde{\eta}_{i}}{\sigma_{x, i}^{2}}\left(x_{i}-\bar{x}\left(z_{i}\right)\right)-\frac{\rho\left(z_{i}\right)}{\sigma_{x, i} \sigma_{m, i}} \tilde{q}_{i}\left(x_{i}-\bar{x}\left(z_{i}\right)\right)\right\}=0 \\
& \sum_{i} \frac{1}{1-\rho^{2}\left(z_{i}\right)}\left\{\frac{\tilde{q}_{i}}{\sigma_{m, i}^{2}}\left(m_{i}-\bar{m}\left(z_{i}\right)\right)-\frac{\rho\left(z_{i}\right)}{\sigma_{x, i} \sigma_{m, i}} \tilde{\eta}_{i}\left(m_{i}-\bar{m}\left(z_{i}\right)\right)\right\}=0 .
\end{aligned}
$$

$\bar{x}, \bar{m}$ do not correspond to the true mean intrinsic quantities, since only galaxies passing cuts are used to measure them. We can then write

$$
\begin{aligned}
& \bar{a}(z)=\left(\sum_{i} w_{a, i}\right)^{-1} \sum_{i} w_{a, i} a_{i}, \\
& w_{x, i}=\frac{1}{1-\rho^{2}\left(z_{i}\right)}\left\{\frac{\tilde{\eta}_{i}}{\sigma_{x, i}^{2}}-\frac{\rho\left(z_{i}\right)}{\sigma_{x, i} \sigma_{m, i}} \tilde{q}_{i}\right\} \\
& w_{m, i}=\frac{1}{1-\rho^{2}\left(z_{i}\right)}\left\{\frac{\tilde{q}_{i}}{\sigma_{m, i}^{2}}-\frac{\rho\left(z_{i}\right)}{\sigma_{x, i} \sigma_{m, i}} \tilde{\eta}_{i}\right\},
\end{aligned}
$$

where the sum runs over all galaxies passing cuts in the redshift slice.

In order to determine $\varepsilon_{x}, \varepsilon_{m}$, we repeat the measurement of $\bar{x}, \bar{m}$ with magnitude and size cuts varied by $\pm \Delta m, \pm \Delta x$, respectively, where $\Delta m=q\left(m_{\text {cut }}\right) \kappa_{0}$ and $\Delta x=\eta\left(d_{\text {cut }}\right) \kappa_{0}$, and we take $\kappa_{0}=0.02$ (the value of $\varepsilon_{i}$ does not depend significantly on $\left.\kappa_{0}\right)$. Then,

$$
\begin{aligned}
\varepsilon_{x}(z) & =\frac{\bar{x}_{+}(z)-\bar{x}_{-}(z)}{2 \kappa_{0}} \\
\varepsilon_{m}(z) & =\frac{\bar{m}_{+}(z)-\bar{m}_{-}(z)}{2 \kappa_{0}}
\end{aligned}
$$

where $\bar{a}_{ \pm}$indicate quantities measured when using cuts $m_{\text {cut }} \pm$ $\Delta m$ and $x_{\text {cut }} \pm \Delta x$. In order to take into account interdepen- 

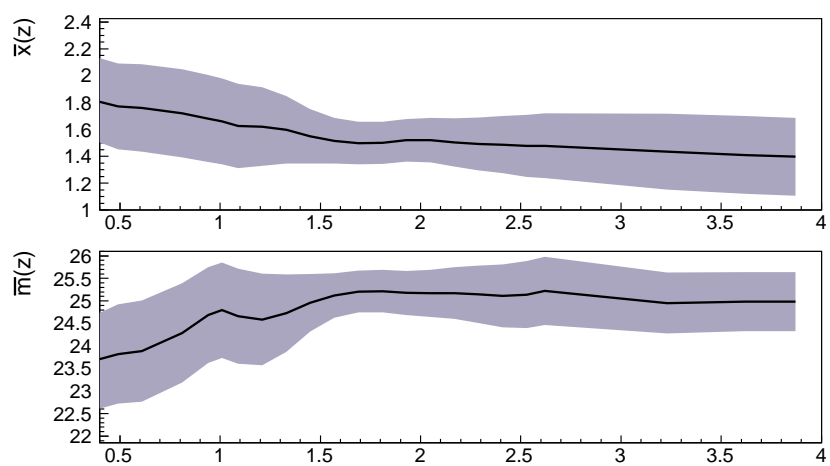

FIG. 1.- Upper panel: Average source galaxy $\log$ size $\bar{x}(z)$ as a function of redshift, measured using Eq. (20). The shaded band shows $\sigma_{x, \text { int }}$. Lower panel: Same, for source galaxy magnitudes $\left(\bar{m}(z), \sigma_{m, \text { int }}\right)$.

dencies, we iterate the measurement of $\bar{x}(z), \bar{m}(z)$ until convergence.

\section{DATA}

Our data set consists of the HST ACS imaging of the COSMOS field (Koekemoer et al. 2007), from which we take the galaxy sizes $d$ (in pixels of 0.03") and magnitudes $m$ in the F814W band. For the size $d$ we use the variable-width Gaussian-filtered second moment of Rhodes et al. (2000), while magnitudes $m$ are MAG_AUTO from SExtractor (Bertin \& Arnouts 1996). We leave more sophisticated, optimized size and flux estimators for future work. The details of the reduction pipeline are described in Leauthaud et al. (2007). The size estimator is PSF-deconvolved, and we thus expect systematic effects of PSF variations to be negligible. In order to avoid any contamination by a background-dependence of the estimated sizes, we only use sky regions within the lower peak (mean pixel background $<0.0062$ ) of the essentially bimodal background distribution in the COSMOS ACS data, comprising $\sim 75 \%$ of the field (see Fig. 8 in Leauthaud et al. (2007)).

For our source galaxy sample, we exclude galaxies in masked regions (Ilbert et al. 2009) and apply the cuts on size, magnitude and redshift summarized in Tab. 1. The size cut approximately corresponds to a measured size (before PSF deconvolution) of 1.4 times the PSF size. Note that in the shear analysis, a significantly stricter cut $(d>3.6)$ is employed. The magnitude cut is mostly determined by the reliability of the photometric redshifts. Finally, the redshift cut excludes a small portion of the sample which does not contribute significantly to the lensing signal-to-noise. We obtain a sample of $N_{\text {src }}=250,500$ galaxies with a median redshift of 1.22 . In the lensing analysis, we also impose the conditions $z_{i,-}>z_{L}$, $z_{i}>z_{L}+0.2$ for each source-lens pair.

We apply the fitting procedure described in the last section, using overlapping redshift slices of half-width $\Delta z=0.25$ separated by $\Delta z$. Fig. 1 illustrates the measured $\bar{x}(z), \bar{m}(z)$ as well as $\sigma_{x \text {,int }}, \sigma_{m \text {,int }}$. We fix $\rho=-0.6$ to improve the stability of the fit. Allowing $\rho$ to vary does not significantly improve the fit. Fig. 2 shows $\varepsilon_{x}$ and $\varepsilon_{m}$. Note that $\varepsilon_{x}<0$ : the number of faint, small galaxies passing the cuts for $\kappa>0$ reduces the average size, counteracting the lensing effect on the sizes themselves. The analogous holds for magnitudes, where $\varepsilon_{m}>0$. This effect can in principle be avoided by cutting on a quantity not affected by lensing (e.g., surface brightness) instead of magnitude and size. We leave the exploration of this for future work.

While the estimators in Sec. 2 were designed to rely as much as possible on data alone, we do need simulations to de-
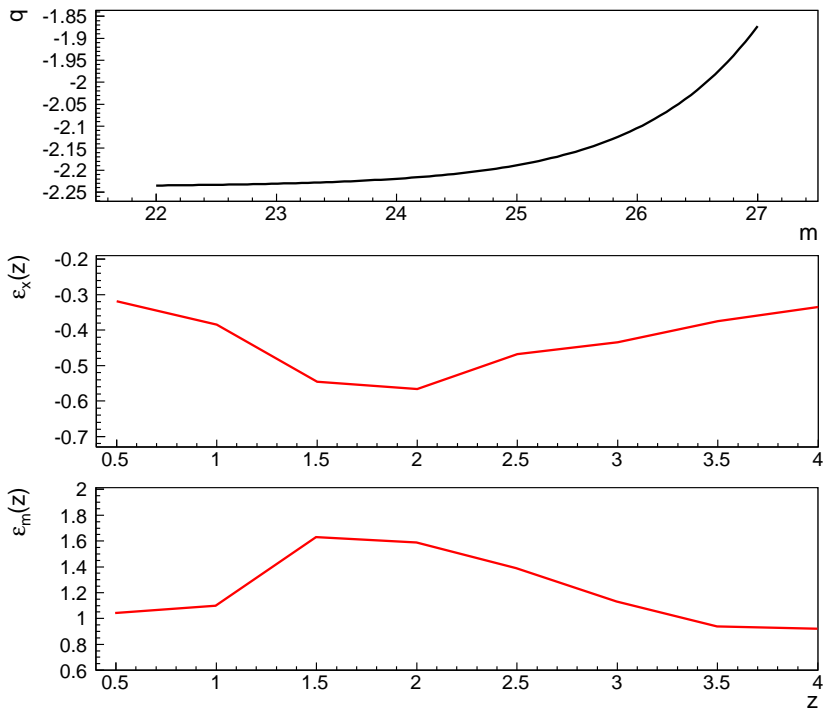

FIG. 2.- From top to bottom: a) Flux lensing efficiency $q$ as a function of magnitude $m . b$ ) Lensing bias correction $\varepsilon_{x}$ for galaxy (log) sizes. The effective size lensing efficiency is given by $\tilde{\eta}=\eta+\varepsilon_{x} . c$ ) Lensing bias effect $\varepsilon_{m}$ for magnitudes. Correspondingly, $\tilde{q}=q+\varepsilon_{m}$.

termine the lensing efficiencies $\eta, q$. To simulate ACS data, we use the simage software package (Massey et al. 2004; Ferry et al. 2008; Dobke et al. 2010), as previously adopted for the Shear TEsting Programme (STEP; Massey et al. 2007) and to calibrate the COSMOS shear analysis (Leauthaud et al. 2007). The parameters were tuned to mimic the galaxy source counts and correlated background noise properties of the reduced and stacked COSMOS images. We model the intrinsic morphologies of source galaxies via shapelets (Refregier 2003a; Massey \& Refregier 2005). We have adapted s image to enable the superposition of an input convergence $\kappa_{\text {sim }}$ and produced images at 15 values of $\kappa_{\text {sim }} \in[-0.2,0.3]$.

To determine how each simulated galaxy responds to magnification, we match galaxies in the lensed and unlensed $\left(\kappa_{\text {sim }}=0\right)$ simulations. We measure the average $x$ and $m$ as a function of the input $\kappa_{\text {sim }}=-0.1 \ldots 0.1$. Then, we estimate $\eta$ through

$$
\eta=\frac{\langle x\rangle_{\kappa_{\mathrm{sim}}=\kappa}-\langle x\rangle_{\kappa_{\mathrm{sim}=0}}}{\kappa},
$$

and correspondingly for $q$. We have found that for galaxies passing cuts, $\eta$ is within $[0.9,1.1]$ and consistent with 1 for the whole sample (recall that we use a PSF-deconvolved size estimator).

For the lens sample, we use X-ray selected groups since these have been well studied using shear (Leauthaud et al. 2010). The entire COSMOS region has been mapped by XMM-Newton, while the central region is covered by Chandra observations (Hasinger et al. 2007; Cappelluti et al. 2009; Elvis et al. 2009). These X-ray data have been used to construct a group catalog containing 211 extended X-ray sources, spanning the range $0<z_{\mathrm{gr}}<1$ (Finoguenov et al. 2007; Leauthaud et al. 2010). Group membership is assigned to galaxies in the COSMOS catalog based on the photometric redshifts

TABLE 1

SUMMARY OF CUTS APPLIED TO THE SOURCE GALAXY SAMPLE.

\begin{tabular}{c|c}
\hline$m$ & $<25.8$ mag \\
$d$ & $>2$ pixels \\
$z$ & $\in[0.5,4]$ \\
\hline
\end{tabular}




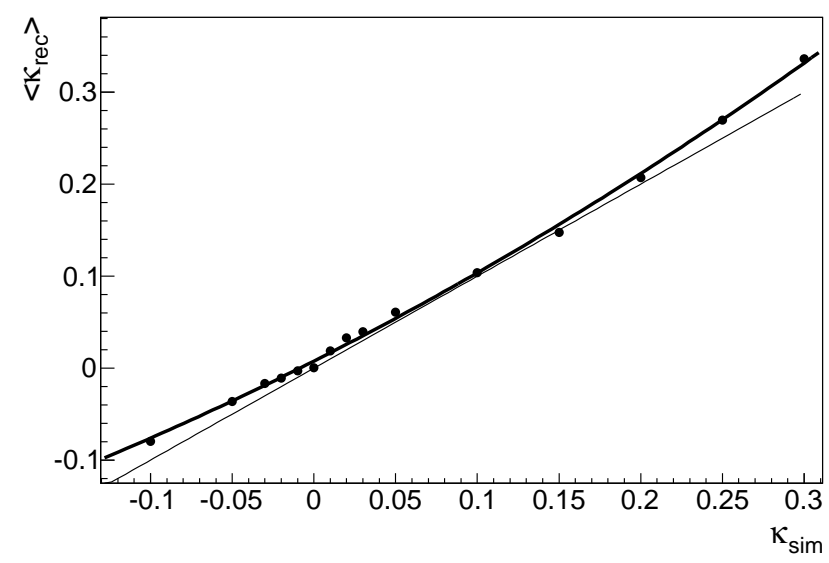

FIG. 3.- Mean reconstructed convergence $\kappa$ in simulations with magnification, as a function of the input $\kappa_{\text {sim }}$. The thin solid line shows the identity while the thick solid line shows a quadratic fit.

and the red sequence. We include only groups with reliable optical associations which have more than 3 members, and we exclude close neighboring systems and groups near the edges of the field or masked regions (FLAG_INCLUDE $=1$ in George et al. (2011a)). We use the location of the most massive group galaxy (MMGG) located within the NFW scale radius of the $\mathrm{X}$-ray centroid as the group center $\left(\mathrm{MMGG}_{\text {scale }}\right.$ as defined in George et al. (2011b)). George et al. (2011b) show that this is likely the most reliable estimate for the group centers. We restrict the redshift range of groups to 0.2-0.6, close to the peak of the lensing sensitivity of the source sample. This selection also reduces the potential impact of catastrophic redshift errors which become more prevalent at $z_{s}>1.5$, and yields $N_{\mathrm{gr}}=61$ groups.

\section{RESULTS}

Before applying our estimators to data, we performed the calibration described in Sec. 2 on the unlensed simulations, and applied the estimators to the lensed simulations as a consistency check. Since the simulations do not contain redshift information, we set $F\left(z_{i}\right)=1$. Fig. 3 shows the mean reconstructed $\kappa$ as a function of the true $\kappa_{\text {sim }}$, along with a quadratic fit,

$$
\left\langle\kappa_{\text {rec }}\right\rangle=p_{0}+p_{1} \kappa_{\text {sim }}+p_{2} \kappa_{\text {sim }}^{2} .
$$

Note that we expect some quadratic correction in this relation, since our estimators neglect all second- and higher-order terms. We found $p_{1}=0.95 \pm 0.05$, indicating that our estimator is close to unbiased, and $p_{2} \approx 0.6$.

Turning to data, we collect source galaxies in bins of physical transverse radius $r$ around the $N_{\text {gr }} \mathrm{X}$-ray groups, and apply the estimator for $\Sigma$. The second order corrections amount to at most $\sim 10 \%$ at the smallest radii, as shown in Leauthaud et al. (2010), and can be neglected given the errors of this measurement. Since $\bar{x}(z), \bar{m}(z)$ are measured in finite redshift bins without any redshift weighting while we apply redshift weighting in the $\Sigma$ measurement, we need to subtract a constant offset $\Sigma_{\text {off }}$ from the measurement. $\Sigma_{\text {off }}$ is measured by pairing all galaxies passing cuts in the COSMOS field with each group, yielding $\Sigma_{\text {off }}=151.9 M_{\odot} / \mathrm{pc}^{2}$. This is equivalent to estimating $\langle\Sigma(r)\rangle_{\text {groups }}-\langle\Sigma\rangle_{\text {COSMOS }}$, where both averages employ the same redshift weighting. Note that a circle of $r \sim 1 \mathrm{Mpc}$ drawn around each group covers a significant fraction of the COSMOS field; thus, we necessarily subtract some of the signal in this way. If we instead restrict the source

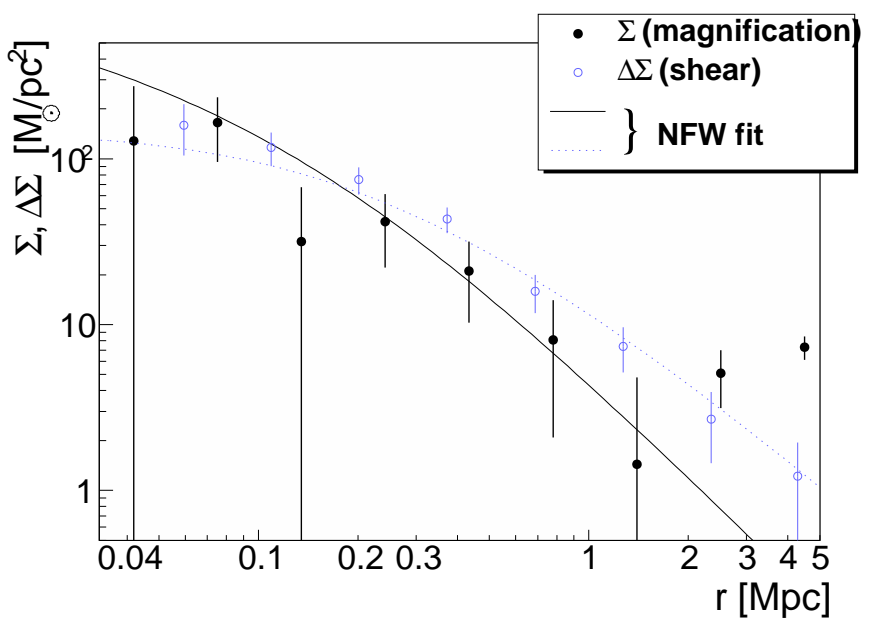

FIG. 4.- Projected surface mass density $\Sigma$ (filled circles) measured around groups using the estimator described in Sec. 2. The open circles show $\Delta \Sigma$ measured using shear for the same group sample. The solid and dotted lines show $\Sigma$ and $\Delta \Sigma$, respectively, for the joint best-fitting NFW model $\left(\lg \left(M / M_{\odot}\right)=13.43 ; r<1 \mathrm{Mpc}\right)$. Note that associated large-scale structure contributes significantly to $\Sigma(r)$ for $r \gtrsim 1 \mathrm{Mpc}$.

sample used for the $\Sigma_{\text {off }}$ estimate to galaxies separated by at least $1 \mathrm{Mpc}$ in physical transverse distance from each group in the sample, $\Sigma_{\text {off }}$ decreases by $4.4 M_{\odot} / \mathrm{pc}^{2}$, thus increasing $\Sigma(r)$ uniformly by this amount.

The measured $\Sigma(r)$ is shown in Fig. 4. The error bars are those returned by the Gaussian estimators. Measurements of the variance of $\hat{\Sigma}$ applied to random sets of source galaxies indicate that these errors are correct to within $\sim 15 \%$. For $r \lesssim 1 \mathrm{Mpc}$, the errors for different radial bins are independent.

We fit an NFW profile (Navarro et al. 1997) with fixed concentration $c=4$ to the measurement, calculating $\Sigma(r)$ as described e.g. in Schmidt \& Rozo (2011). Furthermore, we restrict the fitting to $r<1 \mathrm{Mpc}$, beyond which the contribution from associated large-scale structure becomes important (see below). This yields a best-fit mass of $\lg \left(M / M_{\odot}\right)=13.25 \pm$ 0.16 (statistical error), corresponding to a detection significance of $\sim 3.8 \sigma$. This corresponds to $\sim 38 \%$ of the signalto-noise of shear. Note that the precise value also depends on the definition of the offset $\Sigma_{\text {off }}$. The best-fit mass is consistent with the estimate from shear, $\lg \left(M / M_{\odot}\right)=13.49 \pm 0.07$ (Leauthaud et al. (2010); again for $r<1 \mathrm{Mpc}$ ).

In principle, a measurement of $\Sigma$ in addition to $\Delta \Sigma$ can break the mass-concentration degeneracy, since the two quantities have different radial dependencies (Rozo \& Schmidt 2010). Due to the limited signal-to-noise of this first measurement, a joint fit to the $\Sigma$ measurement together with $\Delta \Sigma$ from shear only yields an improvement of $\sim 10 \%$ in the area of the error ellipse in the mass-concentration plane. The $\Sigma$ measurements at $r>1 \mathrm{Mpc}$ are consistent with an estimate of the associated large-scale structure contribution (two-halo term) using the halo model, which yields $\Sigma_{2 h} \sim 10 M_{\odot} / \mathrm{pc}^{2}$ with weak $r$ dependence for $r<5 \mathrm{Mpc}$. The corresponding contribution to the shear is much smaller, $\Delta \Sigma_{2 h} \lesssim 1 M_{\odot} / \mathrm{pc}^{2}$, further exemplifying the complementarity of the two measurements. A detailed modeling of these data will be the subject of forthcoming work.

The main systematic uncertainty of the $\Sigma$ measurement is due to the lensing bias effect. We have quantified the impact of the measurement errors on $\varepsilon_{x}, \varepsilon_{m}$ by varying $m_{\text {cut }}, x_{\text {cut }}$, resulting in an overall multiplicative systematic error on $\Sigma$ of 
20\%. Residual effects from PSF and background variations have been found to be negligible. Increasing the minimum size cut from 2 to 3 pixels yields compatible results, as do estimators using sizes and magnitudes separately.

The systematic uncertainty due to photometric redshift errors is similar to that of the shear (Leauthaud et al. (2010); note that we have employed the same source-lens separation criteria as in that paper). Given the lower signal-to-noise of the magnification measurement, this systematic is negligible.

\section{SUMMARY AND DISCUSSION}

We have presented the first measurement of weak lensing magnification using galaxy sizes and fluxes. Measurements of galaxy sizes and magnitudes come for free with weak lensing surveys. We find a signal-to-noise only a factor of $\sim 2-3$ less than shear; thus it is worth pursuing this kind of independent weak lensing measurement. Magnification constitutes a different observable than shear, and the non-local relationship between the two can be used to break degeneracies e.g. when measuring halo profiles.

Furthermore, the systematics affecting this measurement are largely independent from those of the shear: to first order, sizes are only sensitive to the size of the PSF, while magnitudes are PSF-independent. On the other hand, a knowledge of the shape of the PSF is necessary for shear measure- ments. This allows us to use less restrictive cuts. Conversely, a careful calibration of the lensing bias correction is necessary for the magnification measurement, while this effect is not present in the shear at linear order (Schmidt et al. 2009a). Nevertheless, given sufficiently detailed simulations, this is a solvable problem.

While the measurement presented here is limited by signalto-noise and statistics, it is clear that for future, much larger surveys, robust size and flux estimators are essential to a successful application of this method. Given the already promising results, we believe a dedicated effort to develop such estimators for weak lensing will be worthwhile. Finally, our results show that there is significant lensing information in photometric galaxy samples in addition to the shear alone; this should be taken into consideration in the design of future weak lensing experiments.

We thank Jessica Ford, Hendrik Hildebrandt, Eric Huff, Bhuvnesh Jain, Donghui Jeong, Eric Jullo, James Taylor, and Ludovic van Waerbeke for helpful discussions. FS is supported by the Gordon and Betty Moore Foundation. RM is supported by an STFC Advanced Fellowship. JR was supported by JPL, run by Caltech for NASA.

\section{REFERENCES}

Bartelmann, M., \& Narayan, R. 1995, ApJ, 451, 60

Bartelmann, M., \& Schneider, P. 2001, Phys. Rep., 340, 291

Bertin, E., \& Arnouts, S. 1996, A\&AS, 117, 393

Bertin, G., \& Lombardi, M. 2006, ApJ, 648, L17

Cappelluti, N., et al. 2009, A\&A, 497, 635

Croton, D. J., et al. 2005, MNRAS, 356, 1155

Dobke, B. M., Johnston, D. E., Massey, R., High, F. W., Ferry, M., Rhodes, J., \& Vanderveld, R. A. 2010, PASP, 122, 947 Elvis, M., et al. 2009, ApJS, 184, 158

Ferry, M., Rhodes, J., Massey, R., White, M., Coe, D., \& Mobasher, B. 2008, Astroparticle Physics, 30, 65

Finoguenov, A., et al. 2007, ApJS, 172, 182

George, M. R., et al. 2011a, ArXiv e-prints

-. 2011b, in prep.

Hasinger, G., et al. 2007, ApJS, 172, 29

Hildebrandt, H., van Waerbeke, L., \& Erben, T. 2009, A\&A, 507, 683

Hoekstra, H., \& Jain, B. 2008, Annual Review of Nuclear and Particle Science, 58, 99

Huff, E. M., \& Graves, G. J. 2011, ArXiv e-prints

Ilbert, O., et al. 2009, ApJ, 690, 1236

Jain, B. 2002, Astrophys. J., 580, L3

Koekemoer, A. M., et al. 2007, ApJS, 172, 196

Leauthaud, A., et al. 2007, ApJS, 172, 219

-. 2010, ApJ, 709, 97

Maltby, D. T., et al. 2010, MNRAS, 402, 282
Massey, R., Kitching, T., \& Richard, J. 2010, Reports on Progress in Physics, 73, 086901

Massey, R., \& Refregier, A. 2005, MNRAS, 363, 197

Massey, R., Refregier, A., Conselice, C. J., David, J., \& Bacon, J. 2004, MNRAS, 348, 214

Massey, R., et al. 2007, MNRAS, 376, 13

Ménard, B., Scranton, R., Fukugita, M., \& Richards, G. 2010, MNRAS, 405, 1025

Munshi, D., Valageas, P., van Waerbeke, L., \& Heavens, A. 2008, Phys. Rep., 462, 67

Nair, P. B., van den Bergh, S., \& Abraham, R. G. 2010, ApJ, 715, 606

Navarro, J. F., Frenk, C. S., \& White, S. D. M. 1997, ApJ, 490, 493 (NFW)

Refregier, A. 2003a, MNRAS, 338, 35

-. 2003b, ARA\&A, 41, 645

Rhodes, J., Refregier, A., \& Groth, E. J. 2000, ApJ, 536, 79

Rozo, E., \& Schmidt, F. 2010, ArXiv e-prints

Schmidt, F., \& Rozo, E. 2011, ApJ, 735, 119

Schmidt, F., Rozo, E., Dodelson, S., Hui, L., \& Sheldon, E. 2009a, ApJ, 702, 593

-. 2009b, Physical Review Letters, 103, 051301

Schrabback, T., et al. 2010, A\&A, 516, A63+

Scranton, R., et al. 2005, ApJ, 633, 589 